\title{
A dynamic logic for QASM programs
}

\author{
Carlos Tavares ${ }^{1}$ \\ High-Assurance Software Laboratory/INESC TEC, Braga, Portugal, \\ ctavares@inesctec.pt
}

\begin{abstract}
We define a dynamic logic for QASM (Quantum Assembly) programming language, a language that requires the handling of quantum and probabilistic information. We provide a syntax and a model to this logic, providing a probabilistic semantics to the classical part. We exercise it with the quantum coin toss program.
\end{abstract}

Keywords: quantum logic, quantum programming, dynamic logic

\section{Introduction}

The programming languages, calculi, and logics, developed in the course of the past 20 years, for quantum computing have been gaining relevance with the appearance of the first proof-of-concept quantum computers and quantum programming languages. One of such is the Quantum Assembly Language [CBSG17], the quantum circuit specification language in use in the commercially available quantum hardware supplied by IBM, the IBM Q platform [ibm18] (a small example of the language is depicted in figure 1 ).

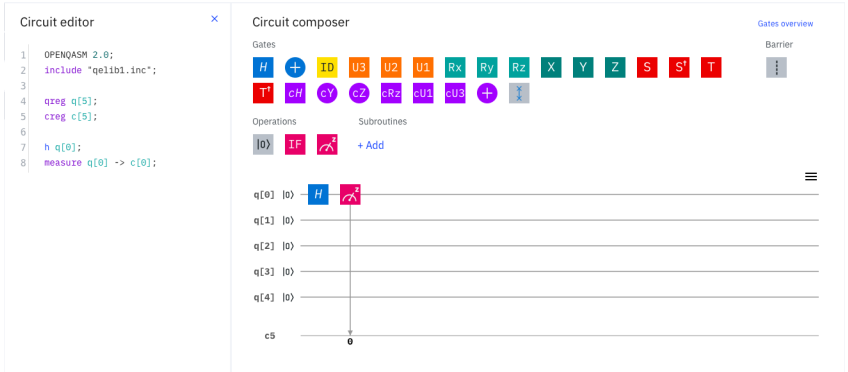

Fig. 1. Example of the definition of a circuit in the QASM language. On the right side the visual definition of the circuit and on the left side the correspondent QASM code.

Besides the description of unitary quantum circuits, the language encompasses classical control flow instructions, such as measurements, which possess a probabilistic nature, and if statements. We propose a dynamic logic for this language exploring two main points of interest: the direct handling of quantum and probabilistic propositions, and a possible axiomatic semantics. 


\section{Quantum computing}

In this section, we introduce quantum computing from a state based perspective (i.e. by the definition of states, transitions, and acceptance states), as usually presented in the literature [Deu85]. For a more complete understanding of quantum computing, we recommend the reading of [NC02].

\section{$2.1 \quad$ States}

The state space of a quantum system is given by the set of unitary vectors (vectors of norm 1) definable in its respective Hilbert space. The qubit, the quantum version of the classical bits, consists of a Hilbert space of dimension $2, \mathcal{H}^{2}$, with $\{|0\rangle,|1\rangle\}$ as an orthogonal basis. The correspondent state space reads as follows:

$$
|\psi\rangle=\alpha|0\rangle+\beta|1\rangle ;|\alpha|^{2}+|\beta|^{2}=1 ; \lambda|\psi\rangle \cong|\psi\rangle, \lambda \in \mathbb{C}
$$

Quantum systems can be combined, employing the tensor product $\otimes$. For a $n$-qubit system, the set of possible states reads as follows:

$$
\bigotimes_{i=0}^{n-1} \mathcal{H}_{i}^{2}
$$

For systems with more than one qubit, one verifies the existence of non-separable states, i.e. states that cannot be written as states of individual qubits, as for instance in the following Bell state: $|\Phi\rangle=\frac{1}{\sqrt{2}}(|00\rangle+|11\rangle)$. The latter is the mathematical expression of the so-called physical phenomenon of entanglement.

\subsection{Transitions (programs)}

In quantum mechanics, transitions preserve unitarity of states. Hence, programs correspond to unitary operators $\left(U \cdot U^{\dagger}=I\right)$. For a quantum system with $n$ qubits the signature of the transition operators reads as follows:

$$
U^{\otimes n}: \mathcal{H}^{2 \otimes n} \rightarrow \mathcal{H}^{2 \otimes n}
$$

In quantum computation practice, a rather less abstract notion is used, the socalled quantum circuits [Deu89], where unitary operators are approximated by compositions of primitive unitary operators, such as the $\mathrm{H}, \mathrm{X}, \mathrm{Y}$, or $\mathrm{Z}$ gates.

\subsection{Acceptance states}

Measurements, (mathematically $\operatorname{Proj}_{\varphi}$, or $|\varphi\rangle\langle\varphi|$ ), can be interpreted as a method that causes the collapse of superposition states to elements of an orthogonal basis, (e.g. in the qubit case $|0\rangle$ and $|1\rangle$ ). An acceptance state is one where the correct output is obtained upon measurement, with probability ${ }^{1}$ greater than $\alpha$.

\footnotetext{
${ }_{1}$ The probability of obtaining $\varphi$ in a measurement is $\left\langle s \mid \operatorname{Proj}_{\varphi} s\right\rangle$ where s is a state and $\langle. \mid$.$\rangle is the internal product of the Hilbert space. In equation (1), |\alpha|^{2}$ and $|\beta|^{2}$, are the probabilities of obtaining $|0\rangle$ and $|1\rangle$, which is 0.5 in both cases: $\left(\left(\frac{1}{\sqrt{2}}\right)^{2}=0.5\right)$.
} 


\section{A dynamic logic for QASM}

The QASM programming language is not a pure quantum programming language as it involves, measurements, which possess a probabilistic nature, and classical flow instructions depending on those measurements, requiring the handling of probabilistic and quantum programs. Our approach to this problem is somehow inspired in the fusion of works of Baltag and Smets [BS04, BBK $\left.{ }^{+} 14\right]$ for the quantum part and of Kozen [Koz85, Koz81] for the probabilistic part.

\subsection{Syntax}

As usual in dynamic logic, the syntax is divided into two layers: one of the programs and one of the formulas. The program's layer encompasses a fragment of the QASM language, which includes the classical control instructions (if statements, creation of classical and quantum registers, and measurements of quantum registers), as well as several standard unitary operations ( $\mathrm{x}, \mathrm{z}, \mathrm{h}$ and cnot gates).

$$
\begin{aligned}
& \langle\text { argument }\rangle \quad::=\text { id | id [index] } \\
& \langle\text { test }\rangle \quad::=\langle\text { argument }\rangle=\langle\text { natural } \text { number }\rangle \\
& \left\langle\pi_{q}\right\rangle \quad::=\mathrm{x} \text { qreg_id [index] | z qreg_id [index] | h qreg_id [index] } \\
& \text { | cx qreg_id [index], qreg_id [index] (unitary gates) } \\
& \text { measure qreg_id } \rightarrow \text { creg_id (measurements) } \\
& \pi_{q} ; \pi_{q} \\
& :=\text { creg id [size] | qreg id [size] (creation of registers) } \\
& \text { | if }\langle\text { test }\rangle \text { then } \pi_{q} \text { (if statements) } \\
& \pi ; \pi \\
& \langle p\rangle \quad::=\perp|\underline{0}| \underline{1} \mid p_{\text {index }}^{\text {register }} \\
& ::=\left(p, f_{\langle t e s t\rangle}=g\right)\left|P^{\geq r} \varphi\right|\langle\pi\rangle \varphi|\neg \varphi| \varphi \vee \varphi \mid \varphi \wedge \varphi
\end{aligned}
$$

Figure 1.1. Formulas Layer and Programs Layer

On the formula side, atomic propositions are pairs $\left(p, f_{\langle t e s t\rangle}=g_{\langle t e s t\rangle}\right)$ where $p$ corresponds to quantum propositions over qubit states and $f_{\langle\text {test }\rangle}=g$ corresponds to equality expressions over the probability distributions definable on the possible tests over classical variables. On the quantum side $\underline{0}$ and $\underline{1}$ denote that 0 or 1 are true upon measurement with 1 as probability, and the $p_{\text {index }}^{\text {register }}$ narrows a proposition range to a specific register and qubit, as for instance $\underline{0}_{0}^{q}$, which means that qubit 0 of register $q$ has value 0 . The $P^{\geq r} \varphi$ modality establishes restrictions to the probability of propositions for instance $P^{=0.5} p$. The $\langle\pi\rangle$ has the usual meaning of "the proposition $\varphi$ may hold upon the execution of program $\pi "$ and the usual minimal set of Boolean connectives is included. 


\subsection{Semantics}

The semantics of this logic is given in terms of a Labelled transition system [HM80], defined by a tuple:

$$
M=\left(\mathcal{G}, \llbracket \cdot \rrbracket: \mathcal{A}_{p} \cup \mathcal{A}_{\pi} \rightarrow 2^{\mathcal{G}} \cup \mathcal{G} \times \mathcal{G}\right)
$$

where $\mathcal{G}$ is a set of states and $\llbracket . \rrbracket$ a meaning function, from the type of the wellformed syntactic expressions of propositions $\left(\mathcal{A}_{p}\right)$ and programs $\left(\mathcal{A}_{\pi}\right)$, to the powerset, and Cartesian product of the set of states, respectively.

\subsection{The state space}

A state of a program in the $Q A S M$ language is defined by its classical and quantum components. Each of such components is divided into one or many independent registers, each composed of a set of quantum or classical bits, resulting in the following state space:

$$
\underbrace{\underbrace{\mathcal{H}^{2} \otimes \ldots \otimes \mathcal{H}^{2}}_{\text {quantum register }} \times \ldots \times \underbrace{\{0,1\} \times \ldots \times\{0,1\}}_{\mathcal{C}} \times \ldots}_{\mathcal{S}}
$$

On the classic side, we work on a probabilistic setting, due to the existence of quantum measurements, which work as random assignments. Thus, the set of possible states corresponds to the distributions definable on the tests ${ }^{2}$ over the classical variables. Therefore, a distribution is given by a measure [Koz85] from the set of tests to the probability interval $[0,1]$ :

$$
\mu_{s}: 2^{\mathcal{C}} \rightarrow[0,1]
$$

However, the actual state in this logic is defined the equality operator over two measures, so an actual state is characterized as a function with signature:

$$
\mu_{s}: 2^{\mathcal{C}} \times 2^{\mathcal{C}} \rightarrow\{0,1\}
$$

In conclusion the state space of a QASM program is given by the Cartesian product of the possible states of the independent quantum and classical registers, denoted Registers, where in the former the set of states is given by the tensor product of quantum bits, and in the latter by the possible distributions definable over the configurations of the classical bits.

$$
\mathcal{G} \equiv \prod_{\text {quantum register } \in \text { Registers }} \bigotimes \mathcal{H}^{2^{\otimes r e g} \text { size }} \times \prod_{\text {classical register } \in \text { Registers }} 2^{2^{\mathcal{C}} \times 2^{\mathcal{C}}}
$$

${ }^{2}$ Tests correspond to the $\sigma$-algebra over the valuation set $\mathcal{C}$. For valuations with a discrete domain, it corresponds to the powerset $2^{\mathcal{C}}$. Tests form a Boolean algebra. 


\subsection{Propositions}

As seen in section 3.1, propositions correspond to a pair of quantum and classical propositions, where quantum propositions are of type $2^{\mathcal{S}}$, the powerset of the quantum state space, and the probabilistic propositions of the type $2^{\mathcal{C} \times \mathcal{C}}$, the pairs of fuzzy predicates ${ }^{3}$ definable on the state space $2^{\mathcal{C} \times \mathcal{C}}$. Therefore, the type of the global propositions reads as follows:

$$
p: 2^{\mathcal{S}} \times\left(2^{\mathcal{C} \times \mathcal{C}}\right)
$$

Definition 1. Semantics for proposition constructors.

We define $\operatorname{proj}_{q}$ as the quantum part of a proposition, and $\operatorname{proj}_{p}$ as the probabilistic part of the proposition.

$i[[\underline{1}]]=\left\{s \mid\left\langle s \mid \operatorname{Proj}_{\underline{1}} s\right\rangle=1\right\}$. Similarly for $[[\underline{0}]]$.

$[[\perp]]-\emptyset$.

$\left[\left[p_{\text {index }}^{\text {register }}\right]-\right.$ The set where the proposition $p$, restricted to a register and a specific qubit index, holds.

ii $\llbracket(p, f=g) \rrbracket=\left\{s \mid s \in \llbracket p \rrbracket \wedge f\left(\operatorname{proj}_{p}(s)\right)=g\left(\operatorname{proj}_{p}(s)\right)\right\}$ and $\operatorname{proj}_{p}(s) \in \mathcal{C}$.

iii $\llbracket P \geq r \varphi \rrbracket=\left\{s \mid\left\langle s \mid \operatorname{Proj}_{\operatorname{proj}_{q} \varphi} s\right\rangle \geq r\right\}$.

The set of states where quantum proposition component $\varphi$ holds with probability greater than $r$.

$i v \llbracket \varphi_{1} \wedge \varphi_{2} \rrbracket=\left\{s \mid s \in \llbracket \operatorname{proj}_{q}\left(\varphi_{1}\right) \cap \operatorname{proj}_{q}\left(\varphi_{2}\right) \rrbracket \wedge s \in \llbracket \operatorname{proj}_{p}\left(\varphi_{1}\right) \cap \operatorname{proj}_{p}\left(\varphi_{2}\right) \rrbracket\right\}$

$v \llbracket \varphi_{1} \vee \varphi_{2} \rrbracket=\left\{s \mid s \in \llbracket \operatorname{proj}_{q}\left(\varphi_{1}\right) \cup \operatorname{proj}_{q}\left(\varphi_{2}\right) \rrbracket \wedge s \in \llbracket \operatorname{proj}_{p}\left(\varphi_{1}\right) \cup \operatorname{proj}_{p}\left(\varphi_{2}\right) \rrbracket\right\}$

$v i \llbracket \neg \varphi \rrbracket=\left\{s \mid s \notin \llbracket \operatorname{proj}_{q} \varphi \rrbracket \wedge s \notin \llbracket \operatorname{proj}_{p} \varphi \rrbracket\right\}$

vii $\llbracket\langle\pi\rangle \varphi \rrbracket=\{s \mid \exists u:(s, u) \in \llbracket \pi \rrbracket \wedge u \in \llbracket \varphi \rrbracket\}$

The set of states where the proposition $\varphi$ holds upon the execution of program

$\pi$.

\subsection{Program semantics}

Programs in this logic correspond to deterministic relations between states:

$$
\llbracket \cdot \rrbracket: \mathcal{A}_{\pi} \rightarrow \mathcal{G} \times \mathcal{G}
$$

This function denotes an accessibility relation, i.e. directed valid transitions between pairs of states (source to output), under the action of a given program.

\footnotetext{
${ }^{3}$ A fuzzy predicate corresponds to a measurable function [Koz85] from the set of states to the probability interval $[0,1]$, in this case, $\mathcal{C} \rightarrow[0,1]$. The fuzzy predicate is characteristic of a test.
} 
Definition 2. Semantics for programs (accessibility relation)

$p \in 2^{\mathcal{S}}$ - any quantum proposition

$\alpha \in 2^{\mathcal{C} \times \mathcal{C}}$ - any probabilistic proposition $\left(f_{\langle t e s t\rangle}=g\right)$

(n) Creation of registers (upon a register is created its value is necessarily 0 , both for quantum and the probabilistic parts):

$\llbracket$ creg reg_id $[$ size $] \rrbracket=\left\{(s, u) \mid s \in \llbracket\left(p, \perp_{\text {reg_id }}\right) \rrbracket \wedge u \in \llbracket\left(p, f_{\text {reg_id=0 }}(u)=1\right) \rrbracket\right\}$

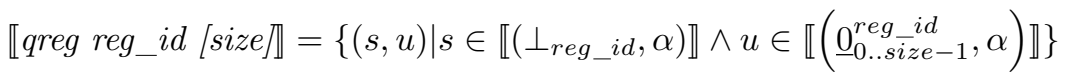

Pairs of states where $\perp$ holds in the source state and 0 in the output state.

(h) Hadamard operator:

$\llbracket h$ reg_id $[$ index] $]=$

$$
\begin{aligned}
& \left\{(s, u) \mid s \in \llbracket\left(\left(P r^{=p_{i}} p\right) \wedge \underline{0}_{i n d e x}^{r e g \_i d}, \alpha\right) \rrbracket \vee s \in \llbracket\left(\left(P r^{=p_{i}} p\right) \wedge \underline{1}_{i n d e x}^{r e g \_i d}, \alpha\right) \rrbracket\right. \\
& \left.\wedge u \in \llbracket\left(P r^{=p_{i} * 0.5}\left(p \wedge \underline{0}_{i n d e x}^{r e g \_i d}\right) \wedge \operatorname{Pr}=p_{i} * 0.5\left(p \wedge \underline{1}_{i n d e x}^{r e g \_i d}\right), \alpha\right) \rrbracket\right\} \\
& \cup\left\{(s, u) \mid s \in \llbracket\left(P r^{=p_{i} * 0.5}\left(p \wedge \underline{0}_{i n d e x}^{r e g \_i d}\right) \wedge P r^{=p_{i} * 0.5}\left(p \wedge \underline{1}_{\text {index }}^{\text {reg_id }}\right), \alpha\right) \rrbracket \wedge\right. \\
& \left.\left(u \in \llbracket\left(\left(P r^{=p_{i}} p\right) \wedge \underline{0}_{i n d e x}^{r e g \_i d}, \alpha\right) \rrbracket \vee u \in \llbracket\left(\left(P r^{=p_{i}} p\right) \wedge \underline{1}_{i n d e x}^{r e g \_i d}, \alpha\right) \rrbracket\right)\right\}
\end{aligned}
$$

Pairs of states defined by either 0 or 1 on the source state and a superposition of 0 and 1 in the output state, or vice-versa.

(x) X operator:

$$
\begin{aligned}
& \llbracket x r e g \_i d[\text { index }] \rrbracket=\left\{(s, u) \mid s \in \llbracket\left(p \wedge \underline{1}_{\text {index }}^{r e g}, \alpha\right) \rrbracket \wedge u \in \llbracket\left(p \wedge \underline{0}_{\text {index }}^{\text {reg_id }}, \alpha\right) \rrbracket\right. \\
& \left.\vee s \in \llbracket\left(p \wedge \underline{0}_{\text {index }}^{r e g}, \alpha\right) \rrbracket \wedge u \in \llbracket\left(p \wedge \underline{1}_{\text {index }}^{r e g \_i d}, \alpha\right) \rrbracket\right\}
\end{aligned}
$$

Pairs of states where 0 holds in the source state and 1 in the output state, or vice-versa (same effect as a classical not gate).

(m) Measure:

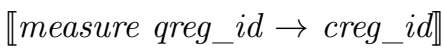

$$
\begin{aligned}
= & \left\{(s, u) \mid s \in \llbracket\left(\bigwedge_{i}^{2^{\text {size }}} P^{=p_{i}} \underline{i}, \mathcal{D}_{\text {creg_id }}\left(\bigwedge_{i} f_{\text {creg_id==i }}\right)\right) \rrbracket\right. \\
& \left.\wedge u \in \llbracket\left(\bigvee_{i} i, \bigwedge_{i} f_{\text {creg_id }==i}(u)==p_{i}\right) \rrbracket\right\}
\end{aligned}
$$

Pairs of states where the probability distribution of the valuations of a set 
of qubits in the source state, is the same as the verified in a set of classical bits in the output state, where $\mathcal{D}_{\text {creg_id }}$ denotes a distribution compatible upon measurement with $\bigwedge_{i} f_{\text {creg_id==i }}(\{d \mid$ meas $\circ d=f\}$ where $\circ$ is the Lebesgue integral)

(;) Sequence $\llbracket \pi_{1} ; \pi_{2} \rrbracket=\left\{(s, u) \mid \exists t(s, t) \in \llbracket \pi_{1} \rrbracket \wedge(t, u) \in \llbracket \pi_{2} \rrbracket\right\}$

\section{An example: A quantum coin tossing program}

This section, illustrates the logic through the proof of correctness of a simple quantum program for quantum coin tossing (prepare a qubit in a superposition state and measure it, obtaining 0 or 1 with equal probability), which translates into the following QASM program:

OPENQASM 2.0;

include "qelib1.inc";

qreg $\mathrm{q}[1]$;

creg $c[1]$;

$\mathrm{h}$ q[0];

measure $q[0] \rightarrow c[0]$;

The correctness of such program implies the following post-condition:

$$
\left(\underline{0}_{0}^{q} \vee \underline{1}_{0}^{q}, f_{\langle c[0]==1\rangle}(x)=0.5 \wedge f_{\langle c[0]==0\rangle}(x)=0.5\right) \text { with } x \in \mathcal{C}
$$

where $\underline{0} \vee \underline{1}$ denotes the quantum qubit $q$ has either, mutually exclusively, the values 0 or 1 , and $\mathcal{C}=\{0,1\}$. The fact that post-condition (6) holds upon the execution of the program qreg $\mathrm{q}[1] ;$ creg c[1]; h q[0]; measure $\mathrm{q}[0] \rightarrow \mathrm{c}[0]$ is expressed through the following formula:

$$
\begin{aligned}
& \langle\text { qreg q[1]; creg c }[1] ; \text { h q }[0] ; \text { measure } \mathrm{q}[0] \rightarrow \mathrm{c}[0]\rangle \\
& \quad\left(\underline{0}_{0}^{q} \vee \underline{1}_{0}^{q}, f_{\langle c[0]==1\rangle}(x)=0.5\right) \wedge\left(\underline{0}_{0}^{q} \vee \underline{1}_{0}^{q}, f_{\langle c[0]==0\rangle}(x)=0.5\right) \text { with } x \in \mathcal{C}
\end{aligned}
$$

This is proved by the rules of section 2 :

Proof.

$\llbracket\langle$ qreg q[1]; creg c[1]; h q[0]; measure q[0] $\rightarrow \mathrm{c}[0]\rangle$

$$
\left(\underline{0}_{0}^{q} \vee \underline{1}_{0}^{q}, f_{\langle c[0]==1\rangle}(x)=0.5 \wedge f_{\langle c[0]==0\rangle}(x)=0.5\right) \rrbracket
$$

$=$

$\{s \mid \exists u:(s, u) \in \llbracket$ qreg q[1]; creg c[1]; h q[0]; measure $\mathrm{q}[0] \rightarrow \mathrm{c}[0] \rrbracket$ 


$$
\begin{aligned}
& \left.\wedge u \in \llbracket\left(\underline{0}_{0}^{q} \vee \underline{1}_{0}^{q}, f_{\langle c[0]==1\rangle}\left(\operatorname{proj}_{p}(u)\right)=0.5 \wedge f_{\langle c[0]==0\rangle}\left(\operatorname{proj}_{p}(u)\right)=0.5\right) \rrbracket\right\} \\
& \text { with } \operatorname{proj}_{p}(u) \in \mathcal{C} \\
& =\text { (use of the }(;) \text { rule) } \\
& \{s \mid \exists u: \exists t:(s, t) \in \llbracket \text { qreg q[1]; creg c[1]; h q }[0] \rrbracket \wedge(t, u) \in \llbracket \text { measure } \mathrm{q}[0] \rightarrow \mathrm{c}[0] \rrbracket \\
& \left.\wedge u \in \llbracket\left(\underline{0}_{0}^{q} \vee \underline{1}_{0}^{q}, f_{\langle c[0]==1\rangle}\left(\operatorname{proj}_{p}(u)\right)==0.5 \wedge f_{\langle c[0]==0\rangle}\left(\operatorname{proj}_{p}(u)\right)=0.5\right) \rrbracket\right\} \\
& =\text { (use of the }(\mathrm{m}) \text { rule) } \\
& \{s \mid \exists u: \exists t:(s, t) \in \llbracket \text { qreg q[1]; creg c[1]; h q[0]』 } \\
& \wedge t \in \llbracket\left(P^{=0.5} \underline{0}_{0}^{q}, P^{=0.5} \underline{1}_{0}^{q}, \mathcal{D}_{c}\left(f_{\langle c[0]==0\rangle} \wedge f_{\langle c[0]==1\rangle}\right)\right) \rrbracket \\
& \left.\wedge u \in \llbracket\left(\underline{0}_{0}^{q} \vee \underline{1}_{0}^{q}, f_{\langle c[0]==1\rangle}\left(\operatorname{proj}_{p}(u)\right)==0.5 \wedge f_{\langle c[0]==0\rangle}\left(\operatorname{proj}_{p}(u)\right)=0.5\right) \rrbracket\right\} \\
& =(\text { use of }(;) \text { and }(\mathrm{h}) . \mathrm{u} \text { can be eliminated because } u \in \llbracket \ldots \rrbracket \text { is true) }
\end{aligned}
$$

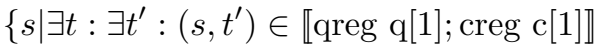

$$
\begin{aligned}
& \wedge\left(t ^ { \prime } \in \llbracket \left(\underline{0}_{0}^{q}, \mathcal{D}_{c}\left(f_{\langle c[0]==0\rangle} \wedge f_{\langle c[0]==1\rangle}\right) \rrbracket \vee t^{\prime} \in \llbracket\left(\underline{1}_{0}^{q}, \mathcal{D}_{c}\left(f_{\langle c[0]==0\rangle} \wedge f_{\langle c[0]==1\rangle}\right) \rrbracket\right)\right.\right. \\
& \wedge t \in \llbracket\left(P^{=0.5} \underline{0}_{0}^{q}, P^{=0.5} \underline{1}_{0}^{q}, \mathcal{D}_{c}\left(f_{\langle c[0]==0\rangle} \wedge f_{\langle c[0]==1\rangle}\right) \rrbracket\right\} \\
& =\text { (use of }(;) \text { and (nreg) rules. } \mathrm{t} \text { can be eliminated because } t \in \llbracket \ldots \rrbracket \text { is true) }
\end{aligned}
$$

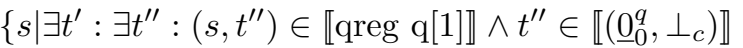

$$
\begin{aligned}
& \wedge\left(t^{\prime} \in \llbracket\left(\underline{0}_{0}^{q}, \mathcal{D}_{c}\left(f_{\langle c[0]==0\rangle} \wedge f_{\langle c[0]==1\rangle}\right) \rrbracket \vee t^{\prime} \in \llbracket\left(\underline{1}_{0}^{q}, \mathcal{D}_{c}\left(f_{\langle c[0]==0\rangle} \wedge f_{\langle c[0]==1\rangle}\right) \rrbracket\right)\right\}\right. \\
& =\text { (use of }(;) \text { and (nreg). t' can be eliminated because } t^{\prime} \in \llbracket \ldots \rrbracket \text { is true) } \\
& \left\{s \mid: \exists t^{\prime \prime}: s \in \llbracket\left(\perp^{q}, \perp^{c}\right) \rrbracket \wedge t^{\prime \prime} \in \llbracket\left(0_{0}^{q}, \perp^{c}\right) \rrbracket\right\} \\
& =\left(\mathrm{t} \text { " can be eliminated because } t^{\prime \prime} \in \llbracket \ldots \rrbracket \text { is true }\right) \\
& \left\{s \mid s \in \llbracket\left(\perp^{q}, \perp^{c}\right) \rrbracket\right\} \text { where } \mathrm{s} \text { is valid state, finishing the proof. }
\end{aligned}
$$

\section{Conclusions}

The paper defined a dynamic logic for a fragment of QASM, combining existent works on dynamic logics for quantum and probabilistic programs and we proved the correctness of a quantum coin toss. However, the logic is still work in progress, being necessary the extension to other examples.

Acknowledgements The author wishes to thank Luís Barbosa and Leandro Gomes, for the useful discussions during the course of this work. The author was funded by an individual grant of reference SFRH/BD/116367/2016, conceded by the FCT - Fundação para a Ciência e Tecnologia under the POCH programme and MCTES national funds. This work was also supported by the KLEE project(POCI01-0145-FEDER-030947-PTDC/CCI-COM/30947/2017), funded by ERDF by the Operational Programme for Competitiveness and Internationalisation, COMPETE2020 Programme and by National Funds through the Portuguese funding agency, FCT. 


\section{References}

$\left[\mathrm{BBK}^{+} 14\right]$ Alexandru Baltag, Jort Bergfeld, Kohei Kishida, Joshua Sack, Sonja Smets, and Shengyang Zhong. Plqp \& company: Decidable logics for quantum algorithms. International Journal of Theoretical Physics, 53(10):3628-3647, 2014.

[BS04] Alexandru Baltag and Sonja Smets. The logic of quantum programs. Proceedings of the 2nd International Workshop on Quantum Programming Languages, pages 39-56, 2004. Available in https://www.mathstat.dal.ca/ selinger/qpl2004/proceedings.html.

[CBSG17] Andrew W Cross, Lev S Bishop, John A Smolin, and Jay M Gambetta. Open quantum assembly language. arXiv preprint arXiv:170\%.03429, 2017.

[Deu85] David Deutsch. Quantum theory, the church-turing principle and the universal quantum computer. Proceedings of the Royal Society of London. A. Mathematical and Physical Sciences, 400(1818):97-117, 1985.

[Deu89] David Elieser Deutsch. Quantum computational networks. Proceedings of the Royal Society of London. A. Mathematical and Physical Sciences, 425(1868):73-90, 1989.

[HM80] Matthew Hennessy and Robin Milner. On observing nondeterminism and concurrency. In Jaco de Bakker and Jan van Leeuwen, editors, Automata, Languages and Programming, pages 299-309, Berlin, Heidelberg, 1980. Springer Berlin Heidelberg.

[ibm18] Ibm q - quantum computing, Jun 2018. Available in: https://www.research.ibm.com/ibm-q/.

[Koz81] Dexter Kozen. Semantics of probabilistic programs. Journal of computer and system sciences, 22(3):328-350, 1981.

[Koz85] Dexter Kozen. A probabilistic pdl. Journal of Computer and System Sciences, 30(2):162-178, 1985.

[NC02] Michael A Nielsen and Isaac Chuang. Quantum computation and quantum information, 2002. 